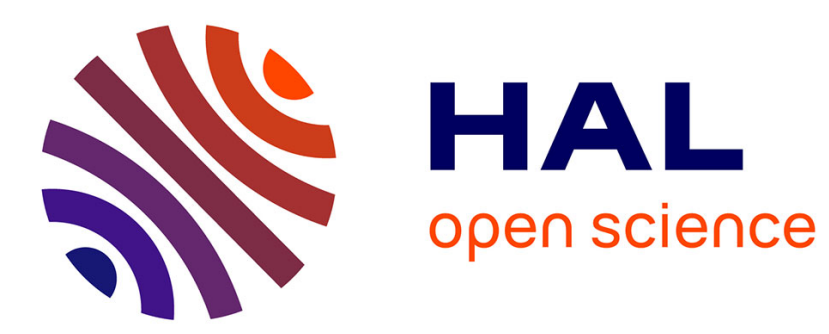

\title{
Coherent Spin Control of Single Molecules on a Surface
} Philip Willke, Tobias Bilgeri, Xue Zhang, Yu Wang, Christoph Wolf, Herve Aubin, Andreas Heinrich, Taeyoung Choi

\section{To cite this version:}

Philip Willke, Tobias Bilgeri, Xue Zhang, Yu Wang, Christoph Wolf, et al.. Coherent Spin Control of Single Molecules on a Surface. ACS Nano, 2021, 15 (11), pp.17959-17965. 10.1021/acsnano.1c06394 . hal-03447976

\section{HAL Id: hal-03447976 https://hal.science/hal-03447976}

Submitted on 24 Nov 2021

HAL is a multi-disciplinary open access archive for the deposit and dissemination of scientific research documents, whether they are published or not. The documents may come from teaching and research institutions in France or abroad, or from public or private research centers.
L'archive ouverte pluridisciplinaire HAL, est destinée au dépôt et à la diffusion de documents scientifiques de niveau recherche, publiés ou non, émanant des établissements d'enseignement et de recherche français ou étrangers, des laboratoires publics ou privés. 


\section{Coherent Spin Control of Single Molecules on a Surface}

Philip Willke, ${ }^{*}, \nabla$ Tobias Bilgeri, ${ }^{\nabla}$ Xue Zhang, Yu Wang, Christoph Wolf, Herve Aubin, Andreas Heinrich,* and Taeyoung Choi*

Cite This: ACS Nano 2021, 15, 17959-17965

Read Online

ACCESS | Lلll Metrics \& More | 回 Article Recommendations | (1) Supporting Information

ABSTRACT: Control of single electron spins constitutes one of the most promising platforms for spintronics, quantum sensing, and quantum information processing. Utilizing single molecular magnets as their hosts establishes an interesting framework since their molecular structure is highly flexible and chemistry-based large-scale synthesis directly provides a way toward scalability. Here, we demonstrate coherent spin manipulation of single molecules on a surface, which we control individually using a scanning tunneling microscope in combination with electron spin resonance. We previously found that iron phthalocyanine $(\mathrm{FePc})$ molecules form a spin-1/2 system when placed on an insulating thin film of magnesium oxide (MgO). Performing Rabi oscillation and Hahn echo measurements, we show that the FePc spin can be coherently manipulated with a phase coherence time $T_{2}^{\text {Echo }}$ of several hundreds of

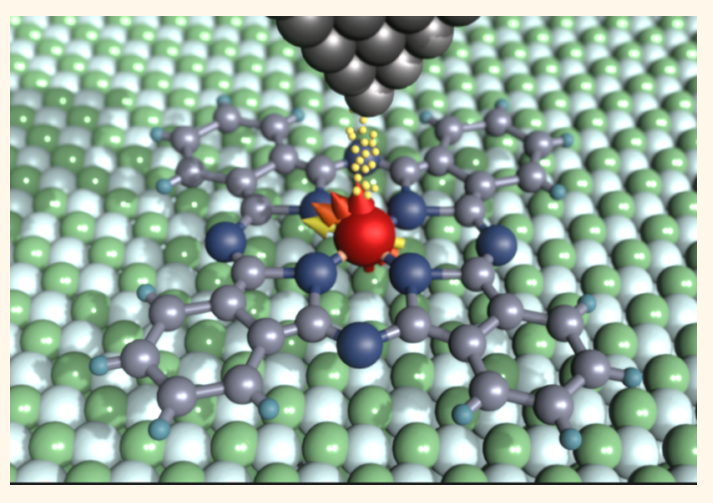
nanoseconds. Tunneling current-dependent measurements demonstrate that interaction with the tunneling electrons is the dominating source of decoherence. In addition, we perform Hahn echo measurements on small self-assembled arrays of FePc molecules. We show that, despite additional intermolecular magnetic coupling, spin resonance and $T_{2}^{\mathrm{Echo}}$ are much less perturbed by $T_{1}$ spin flip events of neighboring spins than by the tunneling current. This will potentially allow for individual addressable molecular spins in self-assemblies and with application for quantum information processing.

KEYWORDS: scanning tunneling microscopy, electron spin resonance, iron phthalocyanine, quantum coherence, spin state, Hahn echo, Rabi oscillations

r he electronic and magnetic properties of single molecular magnets (SMM), which in the simplest case consist of one central metal ion surrounded by ligands, are promising for nanoscale spintronics and future electronic devices. ${ }^{1}$ First, they can be tailored with great precision by their ligand structure, which makes their design highly flexible. Second, they bear the potential of scaling up to very large numbers to form a molecular network while the individual SMM unit remains identical. For the emerging field of quantum information processing, ${ }^{2}$ several techniques including molecular break junctions ${ }^{3,4}$ and ensemble ESR measurements ${ }^{5,6}$ have been used to explore the coherent properties of various SMMs. However, controlling the atomicscale environment and configuration of these molecules in order to study sources of decoherence or to investigate and alter the inter-qubit coupling remained challenging up to now.

Conversely, scanning probe methods (SPM) have been a major tool to study individual molecules on an atomic scale. They allow probing molecular bonding configurations, ${ }^{7}$ as well as the magnetic properties ${ }^{8-16}$ and spin dynamics ${ }^{17}$ of single molecules. Tetrapyrroles, including, for instance, phthalocyanine molecules ${ }^{8,9,11,18}$ and porphyrin complexes, ${ }^{17}$ were intensively studied using scanning tunneling microscopy (STM) and scanning tunneling spectroscopy (STS). They are well-suited for STM experiments due to their simple planar structure, easy preparation, and high stability. In addition, selfassembled networks of metal-organic compounds can easily be realized and investigated. ${ }^{11,13,16,18,19}$ Besides, single molecules have been used in SPM experiments as electric ${ }^{20}$ and magnetic field sensors, ${ }^{21,22}$ and terahertz-assisted STM has granted visualization of coherent molecular vibrations. ${ }^{23}$

Received: July 27, 2021

Accepted: October 13, 2021

Published: November 12, 2021 


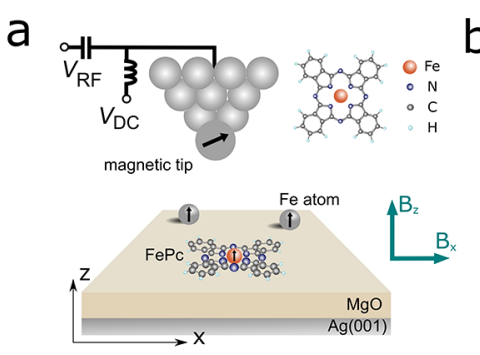

C

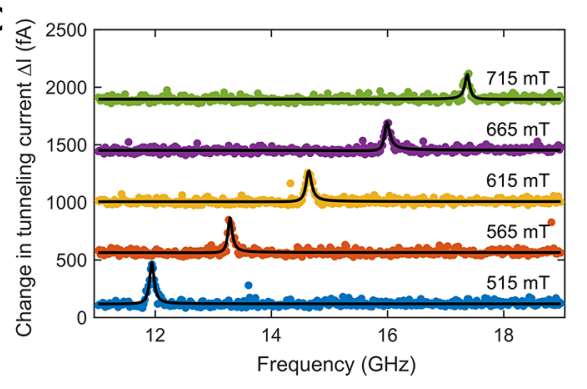

$b$
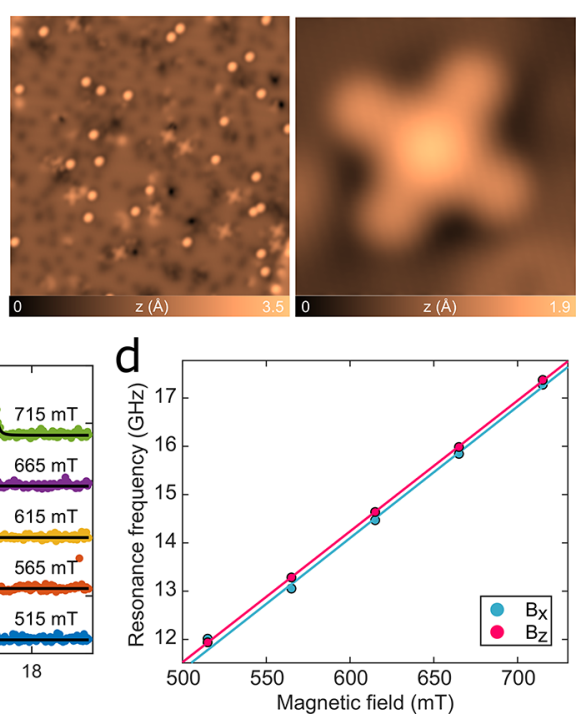

Figure 1. Electron spin resonance (ESR) of individual FePc molecules in a scanning tunneling microscope (STM). (a) Sketch of the experimental setup. Single iron phthalocyanine (FePc) molecules are deposited on two monolayers of $\mathrm{MgO}$ on $\mathrm{Ag}(001)$. Single Fe atoms are additionally deposited to prepare spin-polarized STM tips by transferring the atoms onto the STM tip. An external vector magnet allows adjusting the field in-plane $\left(B_{x}\right)$ and out-of-plane $\left(B_{z}\right)$. In addition to the DC bias voltage $V_{\mathrm{DC}}$, a radio frequency (RF) voltage $V_{\mathrm{RF}}$ at frequency $f$ is applied to the tunnel junction. Inset shows the molecular configuration of FePc. (b) STM topographic image showing several FePc molecules in two orientations $\left(I=20 \mathrm{pA}, V_{\mathrm{DC}}=100 \mathrm{mV}\right.$, image size: $\left.32.5 \mathrm{~nm}\right)$. Right image is a zoom on one of the FePc molecules (I $=50 \mathrm{pA}, V_{\mathrm{DC}}=-5 \mathrm{mV}$, image size: $2.4 \mathrm{~nm}$ ). (c) Continuous-wave ESR spectra taken on an individual FePc molecule at different values of the external $B_{z}$ field $\left(I=27.5 \mathrm{pA}, V_{\mathrm{DC}}=-50 \mathrm{mV}, V_{\mathrm{RF}}=6 \mathrm{mV}, T=0.95 \mathrm{~K}\right)$. Black lines are fits to an asymmetric Lorentzian function. ${ }^{29}$ (d) Resonance frequencies $f_{0}$ as a function of $B_{x}$ and $B_{z}$. Using eq 1 , we extract the magnetic moments of $(0.97 \pm 0.09) \mu_{B}$ along $x$ and $(0.97 \pm$ 0.01) $\mu_{\mathrm{B}}$ along $z$, which are nearly isotropic within the error bars.

Accordingly, controlling the spin properties of a single molecule in a quantum coherent manner as routinely done in other architectures ${ }^{2,4,12}$ would allow establishing a platform to test single molecules for application in quantum information processing. Different approaches of performing electron spin resonance (ESR) on single molecules in an STM have been reported in the last two decades ${ }^{24,25}$ but have not led to further work toward a coherent spin control.

Here, we use electron spin resonance scanning tunneling microscopy (ESR-STM) ${ }^{26}$ to probe the spin properties of single iron phthalocyanine $(\mathrm{FePc})$ molecules on a magnesium oxide $(\mathrm{MgO})$ surface. While in earlier works we investigated the magnetic structure and spin-spin interaction of these molecules using ESR-STM, ${ }^{27}$ we here focus on their coherent spin properties, which, using this technique, was up-to-now only realized for single magnetic adatoms. ${ }^{28}$ It allows us to investigate the spin configuration of the $\mathrm{FePc}$, its spin lifetime, Rabi rate, and phase coherence time. Moreover, we demonstrate that the molecules in self-assembled lattices mostly show the same Hahn echo decay as individual molecules, which promises a straightforward way to realize ESR in large spin lattices with potential use in molecule-based quantum information processing.

\section{RESULTS AND DISCUSSION}

Single Molecule Electron Spin Resonance. Figure 1a illustrates the experimental setup, consisting of individual $\mathrm{FePc}$ molecules adsorbed on $\mathrm{MgO}$ atop a $\mathrm{Ag}(001)$ substrate. In addition, we deposited $\mathrm{Fe}$ atoms, which we used to prepare spin-polarized STM tips ${ }^{26}$ (see Materials and Methods). Figure $1 \mathrm{~b}$ shows an STM topography of several FePc molecules and $\mathrm{Fe}$ atoms on 2 monolayers (ML) of $\mathrm{MgO}$. FePc adsorbs on the oxygen site of $\mathrm{MgO}$ with two possible orientations related by mirror symmetry. ${ }^{27}$ In order to probe the magnetic properties of $\mathrm{FePc}$, we perform tunnel-current-detected ESR by placing the STM tip above the molecule. A radio frequency (RF) voltage $V_{\mathrm{RF}}$ is used here to drive spin transitions in the molecule from the ground state $|0\rangle$ to the excited state $|1\rangle$, which are split by an out-of-plane magnetic field $B_{z}$. This leads to a change in tunneling current $\Delta I$ when the frequency $f$ of $V_{\mathrm{RF}}$ matches the Larmor resonance frequency $f_{0}$ of the FePc Zeeman energy. We show representative ESR spectra for different magnetic fields $B_{z}$ in Figure 1c. By taking continuous wave (CW)-ESR spectra as a function of $B_{z}$, the resonance frequency changes linearly as expected from the resonance condition

$$
h f_{0}=2 \vec{\mu}_{\mathrm{FePc}} \cdot \vec{B}
$$

where $h$ is Planck's constant, $\vec{\mu}_{\mathrm{FePc}}$ is the magnetic moment of FePc, and $\vec{B}$ is the externally applied magnetic field. By performing these experiments for an in- and out-of-plane magnetic field direction $\left(B_{x}\right.$ and $B_{z}$, see Figure $\left.1 \mathrm{~d}\right)$, we find that the slopes of the measurement give magnetic moments of $\mu_{\mathrm{FePc}} \sim 1 \mu_{\mathrm{B}}$. Careful analysis of a large number of data sets and compensation of the tip magnetic field reveals small deviations and a small amount of anisotropy $\left(\mu_{\mathrm{FePc}}^{x, y}=(0.959 \pm 0.010) \mu_{\mathrm{B}}\right.$, $\left.\mu_{\mathrm{FePc}}^{z}=(1.020 \pm 0.023) \mu_{\mathrm{B}}\right) .{ }^{27}$ Nevertheless, we conclude that $\mathrm{FePc}$ molecules constitute in good approximation a spin-1/2 system on $\mathrm{MgO}$. This agrees well with additional density functional theory (DFT) calculations, which suggest that FePc is singly negatively charged as a result of electron charge transfer from the silver substrate to the molecule. ${ }^{27}$ In addition, the DFT calculations showed that the unpaired spin is mostly localized on the central $\mathrm{Fe}$ atom.

Rabi Oscillation Measurements. Next, we access the coherent dynamics of the FePc electron spin by pulsed ESR 
experiments. We here employ the Rabi sequence introduced in ref 28 , that consists of repetitive RF voltage pulses of length $\tau$ with amplitude $V_{\mathrm{RF}}$ and a continuous background of DC voltage $V_{\mathrm{DC}}$ (Figure 2a; see Supporting Information for
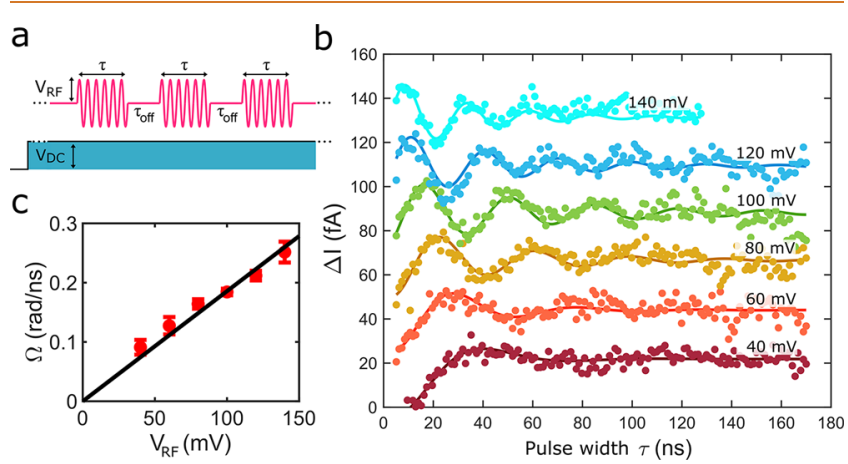

Figure 2. Rabi oscillation measurements. (a) Schematic of the pulse sequence employed for the Rabi measurements. RF pulses of width $\tau$ and amplitude $V_{\mathrm{RF}}$ are applied to the STM tip; a constant DC voltage $V_{\mathrm{DC}}$ was applied during the sequence as an additional spin initialization and readout. (b) Rabi oscillation measurements showing the change in tunneling current $\Delta I$ as a function of $\tau$ for different $V_{\mathrm{RF}}$. Measurements were performed with an open feedback loop $\left(I=5.2 \mathrm{pA}, V_{\mathrm{DC}}=70 \mathrm{mV}, f_{0}=16.227 \mathrm{GHz}, B_{z}=\right.$ $\left.574 \mathrm{mT}, T=390 \mathrm{mK}, \tau_{\text {off }}=700 \mathrm{~ns}\right)$. The measured Rabi oscillations are fitted to $I_{0} \cdot \sin (\Omega \tau+\alpha) \cdot \exp \left(-\frac{\tau}{T_{2}^{\text {Rabi }}}\right)+I_{\text {offset }}$. A linear background stemming from the increasing RF tunnel current for increasing $\tau$ was removed prior to the fitting (see Supplementary Section 1). Note that the pulsed ESR measurements are performed with an out-of-plane magnetic field $B_{z^{*}}$ (c) Extracted Rabi rate $\Omega$ as a function of $V_{\mathrm{RF}}$. The black line is a linear fit to the data with a slope of $(1.86 \pm 0.13) \frac{\mathrm{rad}}{\mu \mathrm{s} \cdot \mathrm{mV}}$.

details). Figure $2 \mathrm{~b}$ shows Rabi oscillation measurements for different $V_{\mathrm{RF}}$. The oscillations become faster for increasing $V_{\mathrm{RF}}$, and we find a linear scaling of the Rabi rate with $V_{\mathrm{RF}}, \Omega \propto V_{\mathrm{RF}}$ (Figure 2c). This effect can be explained by an increasing electric field in the junction for larger $V_{\mathrm{RF}}$, as it is the case for single atoms. ${ }^{26,28,29}$ We here achieve a Rabi rate of $\Omega=(0.25$ $\pm 0.02) \mathrm{rad} / \mathrm{ns}$ for $V_{\mathrm{RF}}=140 \mathrm{mV}$. This leads to a $\pi$-time of $T_{\pi}$ $=\pi / \Omega=(12.6 \pm 1.1) \mathrm{ns}$, the time required for a coherent rotation from the $|0\rangle$ to the $|1\rangle$ state and vice versa. The Rabi oscillations in Figure $2 \mathrm{~b}$ decay in time, so that we fit an exponential decay function $\exp \left(-\frac{\tau}{T_{2}^{\text {Rabi }}}\right)$ with the phase coherence time $T_{2}^{\text {Rabi }}=(42 \pm 15)$ ns. In order to increase the phase coherence time and to further study the sources of relaxation and decoherence in greater detail, we implemented Hahn echo measurements.

Hahn Echo Measurements. Figure 3a shows a typical echo decay using the pulse sequence sketched in the inset. We use $\Omega$ as determined from the Rabi oscillation measurements to create pulses of length $T_{\pi}=\frac{\pi}{\Omega}$ and $T_{\pi / 2}=\frac{\pi}{2 \Omega}$. From exponential decay fits, we find $T_{2}^{\text {echo }}=(196 \pm 26)$ ns (details of the Hahn echo evaluation described in Supplementary Section 2 ). The enhancement compared to $T_{2}^{\text {Rabi }}$ likely stems from the cancellation of low frequency variations in the local magnetic field by the Hahn echo sequence. Tip magnetic field variations induced by minute vibrations in the STM tip position are a likely candidate. ${ }^{28}$ By conducting $T_{2}^{\text {echo }}$ measurements as a function of tunneling current $I$ (Figure $3 \mathrm{~b}$ ), we find that the decoherence time decreases for increasing tunneling current. Figure $3 \mathrm{c}$ shows the decoherence rate $\Gamma_{T 2}=\left(T_{2}^{\text {echo }}\right)^{-1}$ as a function of $I$, which reveals a linear dependence ( $1 \mathrm{pA}$ corresponds to one electron every $160 \mathrm{~ns}$ on average). Evaluating the slope of Figure $3 c$ (see caption) leads to an average decoherence probability of $P_{\mathrm{T} 2}=(32 \pm 3) \%$, meaning that, on average, every third tunnel current electron causes a decoherence event. Moreover, additional $T_{1}$ measurements obtained using a simplified relaxometry pulse sequence (Supplementary Section 3) suggest that these dephasing events are likely connected to $T_{1}$ events, which fully relax the spin state. Thus, the tunneling electrons constitute a dominating source of decoherence and need to be mitigated in order to move toward more advanced pulse sequences.
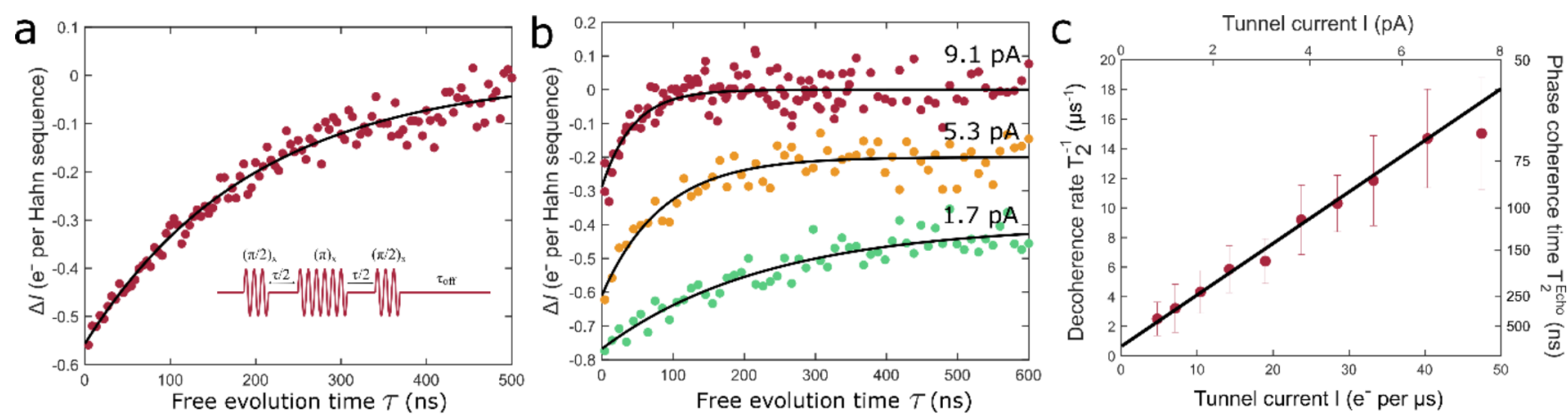

Figure 3. Hahn echo measurements. (a) Representative Hahn Echo measurement. Inset sketches the respective pulse scheme $(I=2.2$ pA, $\left.V_{\mathrm{DC}}=30 \mathrm{mV}, V_{\mathrm{RF}}=100 \mathrm{mV}, f_{0}=16.179 \mathrm{GHz}, T=390 \mathrm{mK}, \tau_{\text {off }}=500 \mathrm{~ns}\right)$. Black line is a fit to $\Delta I=I_{0} \cdot \exp \left(-\frac{\tau}{T_{2}^{\text {Echo }}}\right)$, which here leads to $T_{2}^{\text {Echo }}=(196 \pm 26) \mathrm{ns}$ and $I_{0}=-(0.56 \pm 0.03) e^{-}$per Hahn sequence. $(\mathrm{b})$ Hahn echo taken for different tunneling currents $\left(V_{\mathrm{RF}}=100 \mathrm{mV}\right.$, $B_{z}=574 \mathrm{mT}, f_{0}=16.169 \mathrm{GHz}, T=430 \mathrm{mK}$, data offset for clarity). All data sets were taken at constant tip-sample conductance $G_{\text {set }}=0.076$ $\mathrm{nS}$ to maintain the same tip magnetic field. Different tunneling currents were obtained by changing the bias voltage while the feedback loop was disengaged, maintaining the tip height constant. Extracted values for $T_{2}^{\text {Echo }}: 1.7 \mathrm{pA}:(231 \pm 78) \mathrm{ns} / 5.3 \mathrm{pA}:(84 \pm 22) \mathrm{ns} / 9.1 \mathrm{pA}:(29 \pm$ 10) ns. (c) Decoherence rate $\left(T_{2}^{\mathrm{Echo}}\right)^{-1}$ as a function of tunneling current $I$. Black line is a fit to a simple dephasing model, ${ }^{29} \Gamma_{\mathrm{T} 2}=\left(T_{2}^{\mathrm{Echo}}\right)^{-1}=$ $P_{\mathrm{T} 2} / e \cdot I+1 / T_{2}^{0}$, which assumes a linear decay with the tunneling current. $T_{2}^{0}$ summarizes here all additional contribution to decoherence in the absence of the tunneling current $(I=0 \mathrm{pA})$, and $P_{\mathrm{T} 2}$ is the probability of a dephasing event per tunneling electron. We here find $P_{\mathrm{T} 2}=$ $(32 \pm 3) \%$ and $T_{2}^{0}=(1018 \pm 524)$ ns. 

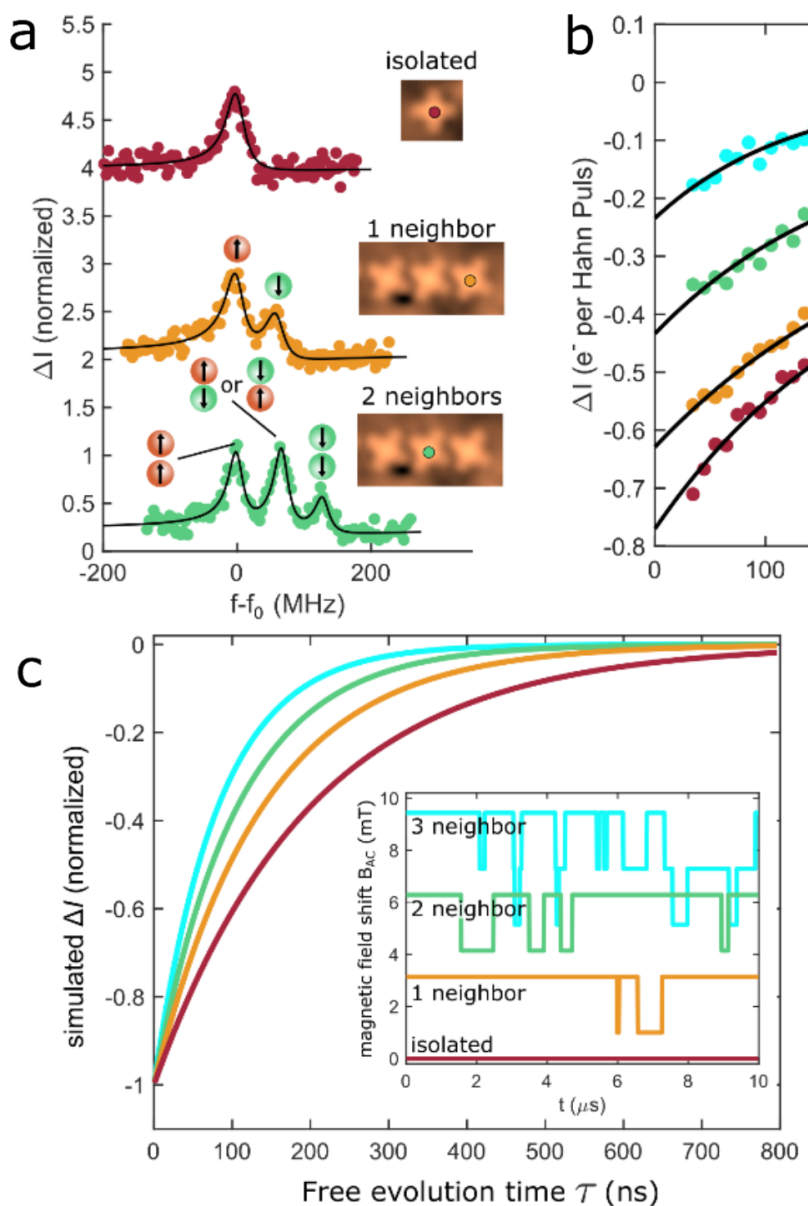
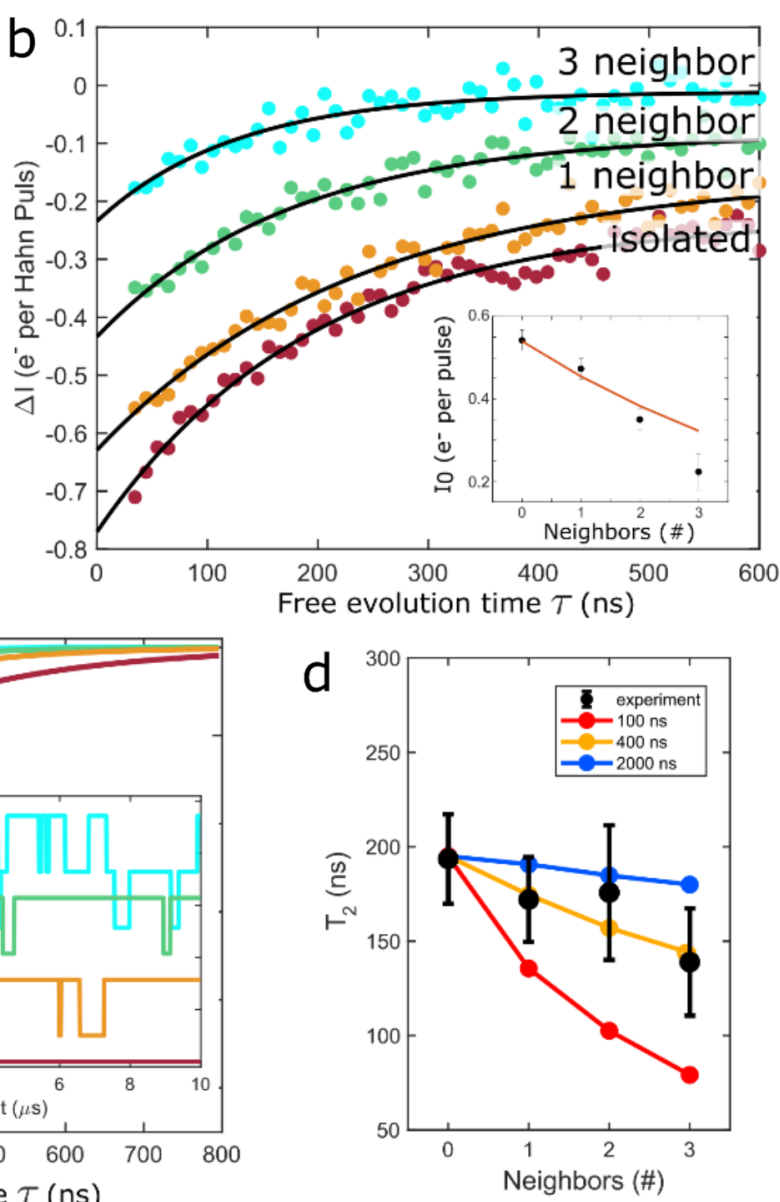

Figure 4. Hahn echo of self-assembled molecules. (a) ESR spectra of molecules with different numbers of neighbors. Each pair is separated by a center-center distance of $1.44 \mathrm{~nm}$ (five MgO lattice constants). Additional peaks in the ESR spectra stem from magnetic coupling to neighboring spins and population of their thermally excited states. Arrows indicate the spin direction of neighboring FePc. $f_{0}(\sim 16 \mathrm{GHz})$ was subtracted to align all spectra $\left(I=3 \mathrm{pA}, V_{\mathrm{DC}}=60 \mathrm{mV}, V_{\mathrm{RF}}=20 \mathrm{mV}, f_{0}=16-16.2 \mathrm{GHz}, T=1 \mathrm{~K}\right)$. (b) Hahn echo measurements comparing molecules in self-assemblies to an isolated molecule. Black lines are a fit to $\Delta I=I_{0} \cdot \exp \left(-\frac{\tau}{T_{2}^{\text {Echo }}}\right)\left(I=2.3 \mathrm{pA}, V_{\mathrm{DC}}=30 \mathrm{mV}, V_{\mathrm{RF}}=\right.$ $100 \mathrm{mV}, f_{0}=16.188 \mathrm{GHz}, T=460 \mathrm{mK}$, data offset for clarity). The inset shows $I_{0}$ (amplitude of the ESR signal) as a function of number of neighbors, and the expected decay is due to thermal population of excited states (topography of the data set for the 3 neighbors in Supplementary Figure S4a). (c) Simulation of the Hahn echo signal in a fluctuating magnetic field $B_{\mathrm{AC}}$ of flipping neighbor spins (simulation parameters: $T_{1}^{\text {neighbor }}=100 \mathrm{~ns}$, $T_{2}^{\text {intrinsic }}=200 \mathrm{~ns}$. Details on the simulation can be seen in Supplementary Section 4). The color scale for different neighbors is the same as in (b). Inset: Example of time traces of simulated $B_{\mathrm{AC}}$ stemming from $T_{1}^{\text {neighbor }}$ at the position of the measured spin. Traces are offset for clarity. (d) $T_{2}^{\text {Echo }}$ as a function of number of neighboring spins for both experiment (black points) and simulation (colored lines, simulated for different values of $T_{1}^{\text {neighbor }}$ ). (Experimental data points are averages over multiple data sets as shown in Supplementary Figure S4b.)

To obtain a tunnel-current free estimate of the coherence time $T_{2}^{0}$, we extrapolate to the limit of vanishing tunneling current in Figure $3 c$, which gives $T_{2}^{0} \sim(1 \pm 0.5) \mu \mathrm{s}$. The sources that will cause decoherence and relaxation in the absence of tunneling current electrons may stem from interaction with the substrate electrons, phonons, and nearby spins on the surface and on the tip, similar to the case of single atomic spins on the same surface. ${ }^{28,29}$ From point contact measurements, we estimate the average time scale for the spin interacting with substrate electrons to be $\sim 1-2 \mu$ s (see Supplementary Section 5), which is in a good agreement with the estimated value of $T_{2}^{0}$.

The highest measured value of $T_{2}^{\text {Echo }}$ for the data set in Figure 3c (399 $\pm 180 \mathrm{~ns})$ exceeds that obtained for hydrated titanium atoms $(\sim 190 \mathrm{~ns} @ 3 \mathrm{pA}) .^{28}$ This is the case despite a 5 times higher point contact conductance for $\mathrm{FePc}$ (Supplementary Section 5), which leads to increased interaction with the substrate electrons. ${ }^{30}$ A likely reason for the higher $T_{2}^{\text {Echo }}$ is here the lower tunneling current reached in our measurement, which, as shown above, has a dominating influence. Compared to most other molecular spin systems studied for quantum information processing, which can reach up to $\sim 3$ orders of magnitude higher values, ${ }^{2,31}$ this time scale still remains short. Also, we would like to highlight that we do not see an effect of the hyperfine coupling to the 4 closest nitrogen atoms in the Hahn echo sequence. From the lowest achieved line width in our CW-ESR spectra $(\sim 25 \mathrm{MHz})$, we can limit the nitrogen hyperfine coupling to $A \leq 10 \mathrm{MHz}$, smaller than values found for, e.g., CuPc in ESR ensemble measurements. ${ }^{5}$ For the latter, the nuclear spin bath limited $T_{2}^{\text {Echo }} \sim 2 \mu \mathrm{s}$. Thus, given our even smaller upper bound for the hyperfine coupling and the more severe influence by the tunnel current electrons, the absence of a dominating decoherence by the nuclear spin bath in our measurements is reasonable. 
Influence of Neighboring Spins. One notable difference compared to most atomic spin centers is the ability of molecules to self-assemble into ordered lattices on the surface. $^{8,9,11,18}$ Figure 4a shows STM topographies of FePc molecules with different numbers of neighbors that naturally arrange in distances with $1.44 \mathrm{~nm}$ spin center spacing, eventually forming a square lattice (Supplementary Figure S4a). In several STM works, the magnetic coupling between neighboring molecules in a lattice was found to be strong $(\sim \mathrm{meV})$, caused by interaction via the substrate electrons $9,11,18,19$ or super-exchange interaction between stacked layers of molecules. ${ }^{16}$ The high resolution of ESR-STM allows us here to observe even the weak intermolecular magnetic coupling of spins on an insulating surface, as evident from the ESR spectra shown in Figure 4a. For these distances, the magnetic interaction has dominant contributions from exchange interaction compared to magnetic dipolar interaction, resulting here in $\Delta f=(64 \pm 2) \mathrm{MHz}$. According to our previous work, ${ }^{27}$ the magnetic dipole interaction contributes here $\Delta f=\frac{\mu_{0}}{\pi(1.44 \mathrm{~nm})^{3} h} \mu_{\mathrm{B}}^{2} \sim 17 \mathrm{MHz}$, since both FePc spins are $\sim 1 \mu_{\mathrm{B}}$. The additional, more dominant contribution arises from exchange interaction between the molecules that is mediated via their ligands. ${ }^{27}$ We also previously observed an avoided level crossing at tunnel current set points about 8-10 pA for magnetic dimers. In this paper, where the measurements are done at $I=3 \mathrm{pA}$, far from the avoided level crossing, the spin states can be described in good approximation as Zeeman basis states $(|\uparrow \uparrow\rangle,|\downarrow \uparrow\rangle,|\uparrow \downarrow\rangle$, and $|\downarrow \downarrow\rangle)$ so that the ESR signals appear at the transition of $|\uparrow \uparrow\rangle$ to $|\downarrow \uparrow\rangle$ and $|\uparrow \downarrow\rangle$ to $|\downarrow \downarrow\rangle$ as shown in Figure 4a.

When the neighboring spins are flipping rapidly through $T_{1}$ processes between their ground and excited state (faster than the time scale of the measurement), their magnetic coupling changes sign and consequently multiple resonances occur in the ESR frequency scan. 32,33

In order to investigate how the spin decoherence is affected by neighboring weakly coupled spins, we perform Hahn echo measurement on the FePc with additional neighbors (Figure $4 \mathrm{~b})$. We find that $T_{2}^{\text {Echo }}$ is only weakly influenced by spin-flip $\left(T_{1}^{\text {neighbor }}\right)$ events of neighboring molecules, also known as spectral spin diffusion, ${ }^{34}$ which affect the phase coherence much less than the tunneling current (Figure 3). A strong dependence on neighboring spins could be expected, if their lifetime $T_{1}^{\text {neighbor }}$ was so short that they would flip on a comparable time scale as the Hahn echo sequence. Simulated Hahn echoes (Figure 4c and Supplementary Section 4), which calculate the influence of stochastically switching neighboring spins, reveal a rapid decay, if a $T_{1}^{\text {neighbor }}$ event happens on the time scale of the pulse sequence $(\sim 100 \mathrm{~ns})$. Since this effect also increases with number of neighbors $n$, we obtain a shortened $T_{2}^{\text {Echo }}$ for Hahn echoes with more neighbors. Figure $4 \mathrm{~d}$ summarized the results of the simulations and compares them to the experimentally obtained $T_{2}^{\text {Echo }}$, averaged over several measurements and molecules (see Supporting Information). The comparison with the experimental data, which shows a slight decrease of $T_{2}^{\text {Echo }}$ with $n$, allows us to estimate the range of $T_{1}^{\text {neighbor }}$ of the remote molecules. Both a short lifetime of $\sim 100 \mathrm{~ns}$ and a long $T_{1}^{\text {neighbor }}$ of $\sim 2 \mu$ s do not fit the experimental data well. Good agreement with the data was obtained in a range of $T_{1}^{\text {neighbor }} \sim 0.4-0.6 \mu \mathrm{s}$ (see Supplementary Section 4). We estimated the intrinsic $T_{1}$ independently from relaxometry, yielding $>0.3 \mu$ s (see
Supplementary Section 3). The presence of the tip and substrate shortens and limits $T_{1}$ due to electron scattering from tip electrode and substrate, respectively. ${ }^{30}$ This means that our estimation of $T_{1}$ of neighboring molecules that are not exposed to tunnel current electrons $(\sim 0.4-0.6 \mu \mathrm{s})$ should lie between $T_{1}$ from relaxometry $(>0.3 \mu \mathrm{s}$, tip is over the molecule) and substrate-induced scattering time $(\sim 1-2 \mu \mathrm{s}$, Supplementary Section 5), which is indeed the case.

\section{CONCLUSIONS}

These experiments demonstrate that not only is a coherent spin manipulation possible on a single molecule level but also they can be used for sensing neighboring spin dynamics in selfassembled lattices. We believe that such spin lattices on a larger scale could potentially permit one to simulate quantum states of matter ${ }^{35,36}$ in future experiments and to study coupled spin dynamics $^{37}$ using single molecules. Accessing the coherent properties of the individual and self-assembled molecules with atomic-scale control could thus allow for analyzing more complex molecular spin structures with potential application in quantum sensing and quantum information processing.

\section{MATERIALS AND METHODS}

Sample Preparation. All sample preparation was carried out in situ at a base pressure of $<5 \times 10^{-10}$ mbar. The $\operatorname{Ag}(001)$ surface was prepared through several cycles of argon-ion sputtering and annealing through e-beam heating. For $\mathrm{MgO}$ growth, the sample was heated up to $430{ }^{\circ} \mathrm{C}$ and exposed to an $\mathrm{Mg}$ flux for $20 \mathrm{~min}$ in an oxygen environment at $10^{-6} \mathrm{mbar}$, leading to an $\mathrm{MgO}$ coverage of $\sim 50 \%$ and layer thicknesses ranging from 2 to 5 monolayers. $\mathrm{FePc}$ was evaporated onto the sample at room temperature using a homebuilt Knudsen cell at a pressure of $9 \times 10^{-10}$ mbar for $90 \mathrm{~s}$. Electronbeam evaporation of $\mathrm{Fe}$ was carried out for $21 \mathrm{~s}$ onto the cold sample. We determined the thickness of $\mathrm{MgO}$ layers through point-contact measurements on single $\mathrm{Fe}$ atoms. ${ }^{30}$ All experiments were carried out in an Unisoku USM1300 low-temperature scanning tunneling microscopy system equipped with high-frequency cabling and a two-axis vector magnet.

Pulsed Electron Spin Resonance Measurements. Spinpolarized tips were prepared by transferring individual $\mathrm{Fe}$ atoms onto the Ag coated PtIr STM tip. The spin polarization was tested through differential conductance measurements on FePc. Magnetic tips showing a high spin contrast were subsequently tested in continuous-wave (CW) ESR-STM measurements. The RF voltage was applied on the tip side of the junction using an RF generator (Keysight E8257D). The RF voltage was combined with the DC tunnel bias using a Bias tee (Marki Microwave BT-0026). Note that, while the bias voltage was applied to the STM tip, all bias signs were inverted in the paper to follow the conventional definition of bias voltage with respect to the sample bias.

For pulsed ESR measurements, we followed the manipulation scheme introduced in ref 28 . An arbitrary waveform generator (AWG, Tektronix 70002A) gated the output of the RF generator to generate the desired pulsed ESR scheme. We used a digital lock-in amplifier (Stanford Research Systems SR860) to read out the ESR signal using an on/off modulation scheme at $95 \mathrm{~Hz}$. A National Instruments DAQ-Box 6343 was used as a modulation source for the AWG and the lock-in amplifier and to read in the output of the lock-in amplifier. Typically, the acquisition time per data point was $5 \mathrm{~s}$ during which the feedback loop was disengaged. Before each data point, the feedback loop was briefly engaged to perform an atom lock sequence in order to correct tip drift. In total, these measurements took $\sim 12 \mathrm{~s}$ per data point, so that a typical Rabi or Hahn measurement including 3 iterations of 100 points took around $1 \mathrm{~h}$. For the current-dependent measurements in Figure $3 b, c$, we altered the tunnel current by changing the bias voltage once the feedback loop had been disengaged. Consequently, all measurements have been performed 
at constant tunnel conductance and constant tip-atom distance, which, in particular, helped to keep the influence of the tip field constant.

\section{ASSOCIATED CONTENT}

\section{SI Supporting Information}

The Supporting Information is available free of charge at https://pubs.acs.org/doi/10.1021/acsnano.1c06394.

Fitting of Rabi measurements; fitting of Hahn echo measurements; relaxometry measurements; Hahn echo simulations in coupled FePc structures; estimation of substrate scattering (PDF)

\section{AUTHOR INFORMATION}

\section{Corresponding Authors}

Philip Willke - Center for Quantum Nanoscience, Institute for Basic Science (IBS), Seoul 03760, Republic of Korea; Ewha Womans University, Seoul 03760, Republic of Korea; Physikalisches Institut, Karlsruhe Institute of Technology, Karlsruhe 76131, Germany; (1) orcid.org/0000-0002-72158419; Email: philip.willke@kit.edu

Andreas Heinrich - Center for Quantum Nanoscience, Institute for Basic Science (IBS), Seoul 03760, Republic of Korea; Department of Physics, Ewha Womans University, Seoul 03760, Republic of Korea; 이이이.org/0000-00016204-471X; Email: heinrich.andreas@qns.science

Taeyoung Choi - Center for Quantum Nanoscience, Institute for Basic Science (IBS), Seoul 03760, Republic of Korea; Department of Physics, Ewha Womans University, Seoul 03760, Republic of Korea; Email: choi.taeyoung@ qns.science

\section{Authors}

Tobias Bilgeri - Center for Quantum Nanoscience, Institute for Basic Science (IBS), Seoul 03760, Republic of Korea; Ewha Womans University, Seoul 03760, Republic of Korea; Institute of Physics, Ecole Polytechnique Fédérale de Lausanne, Lausanne 1015, Switzerland

Xue Zhang - Center for Quantum Nanoscience, Institute for Basic Science (IBS), Seoul 03760, Republic of Korea; Ewha Womans University, Seoul 03760, Republic of Korea; (1) orcid.org/0000-0002-4868-3600

Yu Wang - Center for Quantum Nanoscience, Institute for Basic Science (IBS), Seoul 03760, Republic of Korea; Ewha Womans University, Seoul 03760, Republic of Korea; ○ orcid.org/0000-0002-4116-0364

Christoph Wolf - Center for Quantum Nanoscience, Institute for Basic Science (IBS), Seoul 03760, Republic of Korea; Ewha Womans University, Seoul 03760, Republic of Korea; (1) orcid.org/0000-0002-9340-9782

Herve Aubin - Centre de Nanosciences et de Nanotechnologies (CNRS), University Paris-Sud, Universités Paris-Saclay, C2N, Palaiseau 91120, France

Complete contact information is available at:

https://pubs.acs.org/10.1021/acsnano.1c06394

\section{Author Contributions}

$\nabla_{\text {These authors contributed equally. }}$

\section{Author Contributions}

P.W., T.B., and T.C. conceived the experiment. P.W., T.B., X.Z., Y.W., H.A., and T.C. carried out the measurements. P.W. and T.B. analyzed the data and wrote the manuscript. A.H. and
T.C. supervised the project. All authors discussed the results and contributed to the manuscript.

Notes

The authors declare no competing financial interest.

\section{ACKNOWLEDGMENTS}

P.W., T.B., X.Z., Y.W., A.H., C.W., and T.C. acknowledge support from the Institute for Basic Science under Grant IBSR027-D1. T.B. acknowledges support from the Swiss National Science Foundation under Project nr. 200020 176932. P.W. acknowledges funding from the Emmy Noether Programme of the DFG (WI5486/1-1) and financing from the Baden Württemberg Foundation Program on Quantum Technologies (Project AModiQuS). T.C. acknowledges financial support from the National Research Foundation of Korea 2019R1A4A1029052. We thank T. Esat for writing additional software for controlling the Arbitrary Waveform Generator.

\section{REFERENCES}

(1) Aradhya, S. V.; Venkataraman, L. Single-Molecule Junctions beyond Electronic Transport. Nat. Nanotechnol. 2013, 8 (6), 399.

(2) Gaita-Ariño, A.; Luis, F.; Hill, S.; Coronado, E. Molecular Spins for Quantum Computation. Nat. Chem. 2019, 11 (4), 301.

(3) Thiele, S.; Balestro, F.; Ballou, R.; Klyatskaya, S.; Ruben, M.; Wernsdorfer, W. Electrically Driven Nuclear Spin Resonance in Single-Molecule Magnets. Science 2014, 344 (6188), 1135-1138.

(4) Godfrin, C.; Ferhat, A.; Ballou, R.; Klyatskaya, S.; Ruben, M.; Wernsdorfer, W.; Balestro, F. Operating Quantum States in Single Magnetic Molecules: Implementation of Grover's Quantum Algorithm. Phys. Rev. Lett. 2017, 119 (18), 187702.

(5) Warner, M.; Din, S.; Tupitsyn, I. S.; Morley, G. W.; Stoneham, A. M.; Gardener, J. A.; Wu, Z.; Fisher, A. J.; Heutz, S.; Kay, C. W.; Aeppli, G. Potential for Spin-Based Information Processing in a ThinFilm Molecular Semiconductor. Nature 2013, 503 (7477), 504-508.

(6) Shiddiq, M.; Komijani, D.; Duan, Y.; Gaita-Ariño, A.; Coronado, E.; Hill, S. Enhancing Coherence in Molecular Spin Qubits via Atomic Clock Transitions. Nature 2016, 531 (7594), 348-351.

(7) Gross, L.; Mohn, F.; Moll, N.; Liljeroth, P.; Meyer, G. The Chemical Structure of a Molecule Resolved by Atomic Force Microscopy. Science 2009, 325 (5944), 1110-1114.

(8) Krull, C.; Robles, R.; Mugarza, A.; Gambardella, P. Site- and Orbital-Dependent Charge Donation and Spin Manipulation in Electron-Doped Metal Phthalocyanines. Nat. Mater. 2013, 12 (4), 337-343.

(9) Mugarza, A.; Krull, C.; Robles, R.; Stepanow, S.; Ceballos, G.; Gambardella, P. Spin Coupling and Relaxation inside MoleculeMetal Contacts. Nat. Commun. 2011, 2 (1), 490.

(10) Abdurakhmanova, N.; Tseng, T. C.; Langner, A.; Kley, C. S.; Sessi, V.; Stepanow, S.; Kern, K. Superexchange-Mediated Ferromagnetic Coupling in Two-Dimensional Ni-TCNQ Networks on Metal Surfaces. Phys. Rev. Lett. 2013, 110 (2), 027202.

(11) Franke, K. J.; Schulze, G.; Pascual, J. I. Competition of Superconducting Phenomena and Kondo Screening at the Nanoscale. Science 2011, 332 (6032), 940-944.

(12) Warner, B.; El Hallak, F.; Prüser, H.; Sharp, J.; Persson, M.; Fisher, A. J.; Hirjibehedin, C. F. Tunable Magnetoresistance in an Asymmetrically Coupled Single-Molecule Junction. Nat. Nanotechnol. 2015, 10 (3), 259.

(13) Fu, Y.-S.; Ji, S.-H.; Chen, X.; Ma, X.-C.; Wu, R.; Wang, C.-C.; Duan, W.-H.; Qiu, X.-H.; Sun, B.; Zhang, P.; Jia, J.-F.; Xue, Q.-K. Manipulating the Kondo Resonance through Quantum Size Effects. Phys. Rev. Lett. 2007, 99 (25), 256601.

(14) Kezilebieke, S.; Žitko, R.; Dvorak, M.; Ojanen, T.; Liljeroth, P. Observation of Coexistence of Yu-Shiba-Rusinov States and Spin-Flip Excitations. Nano Lett. 2019, 19 (7), 4614-4619.

(15) Tsukahara, N.; Noto, K. I.; Ohara, M.; Shiraki, S.; Takagi, N.; Takata, Y.; Miyawaki, J.; Taguchi, M.; Chainani, A.; Shin, S.; Kawai, 
M. Adsorption-Induced Switching of Magnetic Anisotropy in a Single Iron(II) Phthalocyanine Molecule on an Oxidized Cu (110) Surface. Phys. Rev. Lett. 2009, 102 (16), 167203.

(16) Chen, X.; Fu, Y. S.; Ji, S. H.; Zhang, T.; Cheng, P.; Ma, X. C.; Zou, X. L.; Duan, W. H.; Jia, J. F.; Xue, Q. K. Probing Superexchange Interaction in Molecular Magnets by Spin-Flip Spectroscopy and Microscopy. Phys. Rev. Lett. 2008, 101 (19), 197208.

(17) Heinrich, B. W.; Braun, L.; Pascual, J. I.; Franke, K. J. Protection of Excited Spin States by a Superconducting Energy Gap. Nat. Phys. 2013, 9 (12), 765-768.

(18) Tsukahara, N.; Shiraki, S.; Itou, S.; Ohta, N.; Takagi, N.; Kawai, M. Evolution of Kondo Resonance From a Single Impurity Molecule to the Two-Dimensional Lattice. Phys. Rev. Lett. 2011, 106 (18), 187201.

(19) Girovsky, J.; Nowakowski, J.; Ali, M. E.; Baljozovic, M.; Rossmann, H. R.; Nijs, T.; Aeby, E. A.; Nowakowska, S.; Siewert, D.; Srivastava, G.; Wäckerlin, C.; et al. Long-Range Ferrimagnetic Order in a Two-Dimensional Supramolecular Kondo Lattice. Nat. Commun. 2017, 8 (1), 15388.

(20) Wagner, C.; Green, M. F.; Maiworm, M.; Leinen, P.; Esat, T.; Ferri, N.; Friedrich, N.; Findeisen, R.; Tkatchenko, A.; Temirov, R.; Tautz, F. S. Quantitative Imaging of Electric Surface Potentials with Single-Atom Sensitivity. Nat. Mater. 2019, 18 (8), 853-859.

(21) Verlhac, B.; Bachellier, N.; Garnier, L.; Ormaza, M.; Abufager, P.; Robles, R.; Bocquet, M. L.; Ternes, M.; Lorente, N.; Limot, L. Atomic-Scale Spin Sensing with a Single Molecule at the Apex of a Scanning Tunneling Microscope. Science 2019, 366 (6465), 623-627.

(22) Czap, G.; Wagner, P. J.; Xue, F.; Gu, L.; Li, J.; Yao, J.; Wu, R.; Ho, W. Probing and Imaging Spin Interactions with a Magnetic Single-Molecule Sensor. Science 2019, 364 (6441), 670-673.

(23) Cocker, T. L.; Peller, D.; Yu, P.; Repp, J.; Huber, R. Tracking the Ultrafast Motion of a Single Molecule by Femtosecond Orbital Imaging. Nature 2016, 539 (7628), 263-267.

(24) Balatsky, A. V.; Nishijima, M.; Manassen, Y. Electron Spin Resonance-Scanning Tunneling Microscopy. Adv. Phys. 2012, 61 (2), $117-152$.

(25) Müllegger, S.; Tebi, S.; Das, A. K.; Schöfberger, W.; Faschinger, F.; Koch, R. Radio Frequency Scanning Tunneling Spectroscopy for Single-Molecule Spin Resonance. Phys. Rev. Lett. 2014, 113 (13), 133001.

(26) Baumann, S.; Paul, W.; Choi, T.; Lutz, C. P.; Ardavan, A.; Heinrich, A. J. Electron Paramagnetic Resonance of Individual Atoms on a Surface. Science 2015, 350, 417-420.

(27) Zhang, X.; Wolf, C.; Wang, Y.; Aubin, H.; Bilgeri, T.; Willke, P.; Heinrich, A.; Choi, T. Electron Spin Resonance of Single Molecules and Magnetic Interaction through Ligands.Nat. Chem. 2021. DOI: 10.1038/s41557-021-00827-7

(28) Yang, K.; Paul, W.; Phark, S. H.; Willke, P.; Bae, Y.; Choi, T.; Esat, T.; Ardavan, A.; Heinrich, A. J.; Lutz, C. P. Coherent Spin Manipulation of Individual Atoms on a Surface. Science 2019, 366 (6464), 509-512.

(29) Willke, P.; Paul, W.; Natterer, F. D.; Yang, K.; Bae, Y.; Choi, T.; Fernández-Rossier, J.; Heinrich, A. J.; Lutz, C. P. Probing Quantum Coherence in Single-Atom Electron Spin Resonance. Sci. Adv. 2018, 4 , eaaq1543.

(30) Paul, W.; Yang, K.; Baumann, S.; Romming, N.; Choi, T.; Lutz, C. P.; Heinrich, A. J. Control of the Millisecond Spin Lifetime of an Electrically Probed Atom. Nat. Phys. 2017, 13 (4), 403-407.

(31) Zadrozny, J. M.; Niklas, J.; Poluektov, O. G.; Freedman, D. E. Millisecond Coherence Time in a Tunable Molecular Electronic Spin Qubit. ACS Cent. Sci. 2015, 1 (9), 488-492.

(32) Choi, T.; Paul, W.; Rolf-Pissarczyk, S.; Macdonald, A. J.; Natterer, F. D.; Yang, K.; Willke, P.; Lutz, C. P.; Heinrich, A. J. Atomic-Scale Sensing of the Magnetic Dipolar Field from Single Atoms. Nat. Nanotechnol. 2017, 12 (5), 420-424.

(33) Yang, K.; Bae, Y.; Paul, W.; Natterer, F. D.; Willke, P.; Lado, J. L.; Ferrón, A.; Choi, T.; Fernández-Rossier, J.; Heinrich, A. J.; Lutz, C. P. Engineering the Eigenstates of Coupled Spin-1/2 Atoms on a Surface. Phys. Rev. Lett. 2017, 119, 227206.
(34) Tyryshkin, A. M.; Tojo, S.; Morton, J. J.; Riemann, H.; Abrosimov, N. V.; Becker, P.; Pohl, H. J.; Schenkel, T.; Thewalt, M. L.; Itoh, K. M.; Lyon, S. A. Electron Spin Coherence Exceeding Seconds in High-Purity Silicon. Nat. Mater. 2012, 11 (2), 143-147.

(35) Khajetoorians, A. A.; Wegner, D.; Otte, A. F.; Swart, I. Creating Designer Quantum States of Matter Atom-By-Atom. Nature Reviews Physics 2019, 1, 703-715.

(36) Choi, D. J.; Lorente, N.; Wiebe, J.; von Bergmann, K.; Otte, A. F.; Heinrich, A. J. Colloquium: Atomic Spin Chains on Surfaces. Rev. Mod. Phys. 2019, 91 (4), 041001.

(37) Veldman, L. M.; Farinacci, L.; Rejali, R.; Broekhoven, R.; Gobeil, J.; Coffey, D.; Ternes, M.; Otte, A. F. Free Coherent Evolution of a Coupled Atomic Spin System Initialized by Electron Scattering. Science 2021, 372 (6545), 964-968.

(38) Willke, P.; Singha, A.; Zhang, X.; Esat, T.; Lutz, C. P.; Heinrich, A. J.; Choi, T. Tuning Single-Atom Electron Spin Resonance in a Vector Magnetic Field. Nano Lett. 2019, 19 (11), 8201-8206. 